\title{
Influence of tunnel length on the pressure wave generated by high-speed trains passing each other
}

\author{
WANG YiWei ${ }^{1 *}$, YANG GuoWei $^{2}$, HUANG ChengGuang ${ }^{1} \&$ WANG Wei ${ }^{3}$ \\ ${ }^{1}$ Key Laboratory for Hydrodynamics and Ocean Engineering, Institute of Mechanics, Chinese Academy of Sciences, Beijing 100190, China; \\ ${ }^{2}$ Key Laboratory of High Temperature Gas Dynamics, Institute of Mechanics, Chinese Academy of Sciences, Beijing 100190, China; \\ ${ }^{3}$ Institute of Mechanics, Chinese Academy of Sciences, Beijing 100190, China
}

Received December 23, 2010; accepted June 29, 2011; published online November 27, 2011

\begin{abstract}
The prediction of the pressure wave amplitude produced when two trains pass each other in the tunnel is important to the train design for airtightness and tunnel conditions in China. In this paper, the key factors of this problem were firstly studied based on theoretical analysis. The equation of the worst tunnel length for the global maximum and minimum pressure values was derived. Then, the influence of tunnel length on global minimum pressure and the critical region in which the global minimum pressure varies rapidly were investigated. Finally, a numerical method based on two-dimensional Navier-Stokes equations was established. Typical conditions of two trains passing-by in tunnels of different lengths were simulated. The theoretical and computational results agree with each other closely.
\end{abstract}

passing in tunnel, air pressure wave, tunnel length, numerical simulation

Citation: Wang Y W, Yang G W, Huang C G, et al. Influence of tunnel length on the pressure wave generated by high-speed trains passing each other. Sci China Tech Sci, 2012, 55: 255-263, doi: 10.1007/s11431-011-4588-5

\section{Introduction}

High-speed trains run as fast as a cruising light aircraft or a taking-off jet plane. The corresponding aerodynamic problems can never be neglected $[1,2]$. Special aerodynamic problems may happen when trains passing each other. In these cases, the aerodynamic performance of high-speed trains differs substantially from that of a single train in the open area. Particularly when the trains pass each other in the tunnel, pressure waves arise when the head and the rear of the train are entering the tunnel. Complicated and strong pressure waves are generated due to propagation, reflection and superposition of the existing waves in the tunnel.

For the design of new high-speed trains, it is crucial to obtain the amplitude of pressure variations on the train

*Corresponding author (email: wangyw@imech.ac.cn) surface when trains run or pass each other in tunnel. Considering the actual development of high-speed railways in China, the cross-section area of the tunnel and the line spacing of the trains have already been prescribed, while the length of the tunnel varies considerably from short, medium, long to specially long ones, in a range from $100 \mathrm{~m}$ to $10 \mathrm{~km}$ or so. Pressure waves differ significantly for different lengths, and it is very difficult to analyze the pressure wave for each tunnel. So that it is very meaningful to investigate the influence of tunnel length on the peak pressure under the actual conditions in China, which can provide a generalized standard for train body design. However, because of the difference of real problems, researchers in foreign countries have carried out little study in this respect. Tian [3], Mei [4] gave qualitative solutions to the aforementioned problem in their books. Wang et al. [5] researched the propagation, reflection and superposition processes of pressure waves 
and showed the formulas and tables of the worst length in single-line tunnels.

On the other hand, the approaches to carry out the study of tunnel aerodynamics are summarized into four classes: theoretical analysis, real vehicle tests $[2,6]$, wind tunnel investigations $[7,8]$ and numerical simulations. Based on the purpose of our study, one-dimensional compressible unsteady flow models, axial symmetrical two-dimensional compressible unsteady turbulence models, and three-dimensional turbulence flow models are utilized, respectively. The characteristic line is the mostly used for one-dimensional analysis [4, 9]. Nonlinear partial differential equations are simplified to hyperbolic equations, and low computational cost is achieved for this approach. It is widely used in solving problems such as trains running, passing each other and determining the effects of auxiliary vertical shafts in the tunnel. With the development of computers and computational technologies, axial symmetrical two-dimensional models [10, 11] and three-dimensional turbulence models with a heavy computational cost are also widely adopted [12-14]. With the acquisition of the flow field of the trains entering the tunnel or passing each other in the tunnel, details of the flow field around the trains can be obtained, including the flow in the cross section of the trains or the aerodynamic forces on special parts of the trains, which are of great importance to the structure and airtightness design of the trains. In addition, other methods are also able to solve the problem above, including the analysis of the effect of tunnel entrance hood in acoustical theory $[15,16]$.

In the present paper, the theoretical analysis is firstly used to deduce the relationship among the worst tunnel length, the critical length region, the length of the train and the running speed. Then in order to verify the relationship, a two-dimensional model is adopted to carry out simulation of trains passing by each other under different tunnel lengths. Finally, the comparison of the numerical results and ana- lytical results are performed to determine the exact formulas mentioned above.

\section{Theoretical analysis}

The case of a train entering the tunnel resembles the piston entering the cylinder, which greatly stagnates the airflow due to obstacle of the tunnel wall. Compression waves arise when the head of the train enters the tunnel and expansion waves emerge when the rear of the train enters the tunnel. The pressure in the tunnel changes due to the propagation of the pressure waves. Reflections on both sides of the tunnel cause the pressure variation much more complicated in the tunnel and on the surface of the train. Assuming two trains entering the tunnel of worst length simultaneously in opposite directions, the greatest superposition of pressure waves will arise and cause the worst aerodynamic loads, based on which we can investigate the design boundaries for the structure strength and airtightness of the train in the worst situations.

\subsection{Influencing factors}

In the condition that trains pass each other in the tunnel, factors greatly affecting the pressure change on the surface of the trains are listed as follows:

1) The initial compression wave when the head of the train enters the tunnel and the expansion wave due to its reflection at the exit (as shown in Figure 1(a) and (c));

2) The initial expansion wave when the rear of the train enters the tunnel and the compression wave due to its reflection at the exit (as shown in Figure 1(b));

3) The surface pressure change when the trains pass each other. Because the pressure on the surface of the train is mainly negative when a single train runs in the tunnel, the surface pressure decreases (as shown in Figure 1(d)).

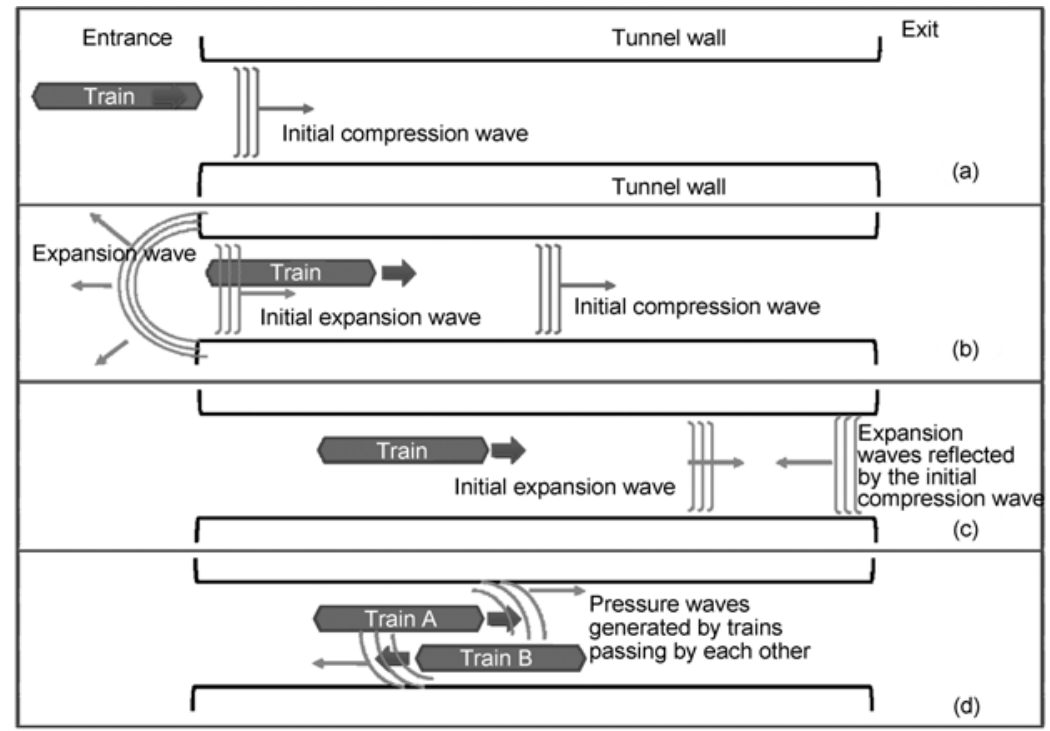

Figure 1 Influencing factors of trains running through and passing each other in the tunnel. 


\subsection{The worst tunnel length}

The pressure waves propagate with the sound speed, and the containing energy dissipates when the waves propagate and reflect in a long tunnel. In short tunnels the dissipation is very small, so much severer pressure variations emerge. However, if the tunnel length is too short, the pressure waves cannot be fully superimposed. So in order to get the lowest valley pressure of different lengths on the surface of the train, the required conditions are that the expansion wave due to the reflection of the initial compression wave generated by the head of train A superimposes exactly with the initial expansion wave generated by the rear of train B, and a stronger expansion wave arises (as shown in Figure 2). Then this stronger expansion wave passes the trains exactly when the trains pass by each other. As long as the conditions above are satisfied, the minimum surface pressure is achieved, so as to obtain the worst tunnel length.

Considering the situation in which two trains with the same length enter the tunnel at the same time in opposite directions, the following equation is obtained:

$$
\frac{L_{t}}{c}=\frac{L_{v}}{v},
$$

where $L_{t}$ is the worst tunnel length, $c$ is the sound speed, $L_{v}$ is the vehicle length, and $v$ is the speed of the trains.

To non-dimensionalize the parameters, the following equation is obtained:

$$
\frac{L_{t}}{L_{v}}=\frac{1}{M}
$$

where $M=v / c$ is the Mach number for the train running.

Besides, about the maximum pressure, the worst tunnel length also satisfies eq. (2). In this condition, when the compression waves generated by train A reach the tunnel exit, the expansion wave is just generated by train B. Consequently, the compression wave can maximize its effect. If the tunnel is shorter, the compression waves have not contributed adequately to get the highest peak pressure. If the tunnel is longer, the compression waves will decay during the propagation.

So for the situation mentioned above, the worst tunnel lengths corresponding to the maximum and minimum surface pressures are the same, which are expressed as a direct relationship between the length of the train and the running Mach number as eq. (2) describes.

\subsection{Critical length region}

Because the negative pressure plays an important role in the passing-by process, the interaction of its primary influence factors (the second and third factors mentioned in section 2.1) need to be analyzed.

As shown in Figure 2, $x$ shows the distance of train rear to the tunnel entrance when the expansion wave reaches the train head by which it was generated. So $x$ can be described as

$$
\frac{x+L_{v}}{c}=\frac{x}{v} \Rightarrow x=\frac{M L_{v}}{1-M} .
$$

About the interaction of factors 2) and 3) in section 2.1, there are several typical lengths as follows.

1) The front of train $A$ is just running out of the tunnel exit exactly when the expansion wave A (generated by train A itself) reaches the front (see Figure 3(a)). In this condition, the tunnel length satisfies

$$
L_{t 1}=L_{v}+x \Rightarrow \frac{L_{t 1}}{L_{v}}=\frac{1}{1-M}
$$

If the tunnel length is less than $L_{t 1}$, because the train body is already locally pulled out of the tunnel, the expansion wave will not fully act on the train body.

2) The front of train A just meets the rear of opposite train B exactly when the expansion wave A (generated by train A itself) reaches the front (see Figure 3(b)). In this condition, the tunnel length satisfies

$$
L_{t 2}=L_{v}+2 x \Rightarrow \frac{L_{t 2}}{L_{v}}=\frac{1+M}{1-M}
$$

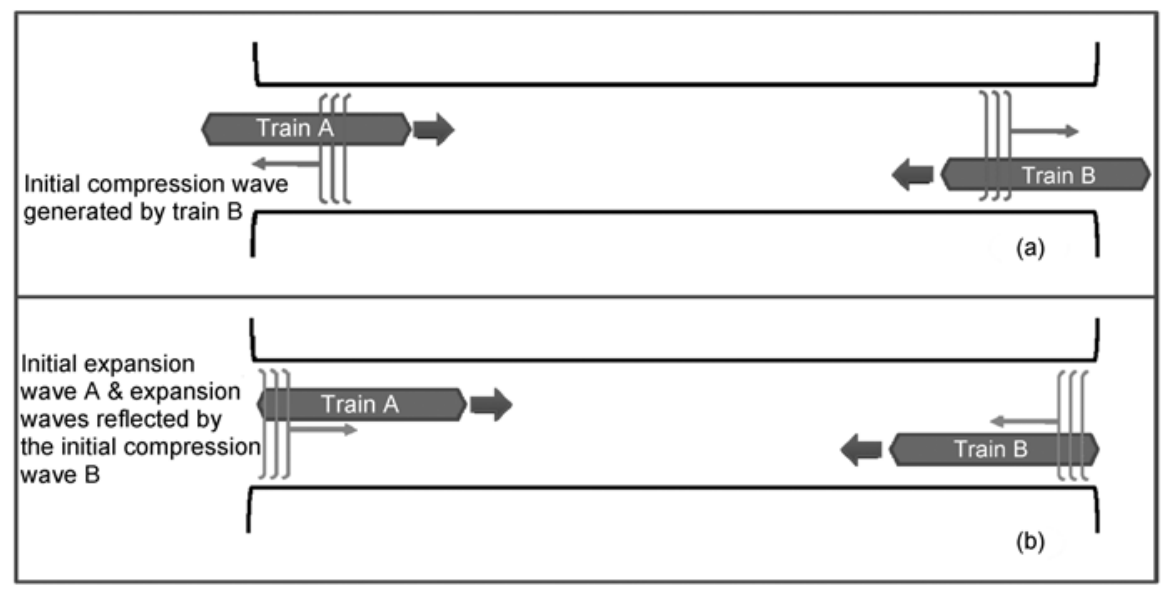

Figure 2 Development of pressure waves in a tunnel with the worst length. 
If the tunnel length is less than $L_{t 2}$, the initial expansion wave cannot superimpose the negative pressure generated by trains passing.

3) The front of train A just meets the front of opposite train B exactly when the expansion wave A (generated by train A itself) reaches the front (see Figure 3(c)). In this condition, the tunnel length satisfies

$$
L_{t 3}=2 L_{v}+2 x \Rightarrow \frac{L_{t 3}}{L_{v}}=\frac{2}{1-M} .
$$

If the tunnel length is greater than $L_{t 3}$, the initial expansion wave has already fully acted on the train body when the two trains pass by each other.

Therefore, if the tunnel length is less than $L_{t 1}$ or $L_{t 2}$ in eqs. (4) and (5), the negative pressure on train surfaces will be less serious. If the tunnel length is greater than $L_{t 3}$ in eq. (6), the situation will become worse. So $\left(L_{t 1}, L_{t 3}\right)$ can be seen as the critical tunnel length region of minimum pressure of trains passing by each other in tunnel.

In summary, considering the reality in China, when two sixteen-coach marshalling trains (about $400 \mathrm{~m}$ long) enter the tunnel (with a cross-section area of $100 \mathrm{~m}^{2}$ ) at the speed of $300 \mathrm{~km} / \mathrm{h}$, the worst tunnel length is $1632 \mathrm{~m}$, and the critical length region is between 529-1060 m (calculated by eqs. (4) and (6)), which is proved in the following sections.

\section{Numerical methods}

Considering the above question, CFD simulations will be used to verify the result in section 2 . Since the calculation of three-dimensional verification is very expensive, a simplified $2 \mathrm{D}$ method is adopted to simulate two-dimensional trains passing through different tunnel lengths. The governing factors are the initial compression wave and expansion wave, which can be well described by $2 \mathrm{D}$ simulation. On the other hand, pressure waves formed while two trains passing by are controlled by the pressure distribution in the horizontal section around the train heads. When the length of train head's profile and the distances between trains are similar, 2D simulations also can approximately capture the passing-by efforts. Consequently, 2D simulation is appropriate for these verification cases.

\subsection{Governing equations}

For simulation of high speed trains, compressible ReynoldsAverage Navier-Stokes equations are utilized as follows:

$$
\frac{\partial \rho}{\partial t}+\frac{\partial}{\partial x_{i}}\left(\rho u_{i}\right)=0
$$

$$
\begin{aligned}
& \frac{\partial}{\partial t}\left(\rho u_{i}\right)+\frac{\partial}{\partial x_{j}}\left(\rho u_{i} u_{j}\right) \\
& =-\frac{\partial p}{\partial x_{i}}+\frac{\partial}{\partial x_{j}}\left[\mu\left(\frac{\partial u_{i}}{\partial x_{j}}+\frac{\partial u_{j}}{\partial x_{i}}-\frac{2}{3} \delta_{i j} \frac{\partial u_{l}}{\partial x_{l}}\right)\right]+\frac{\partial}{\partial x_{j}}\left(-\rho \overline{u_{i}^{\prime} u_{j}^{\prime}}\right) .
\end{aligned}
$$

For closure, the Boussinesq assumption is adopted, taking the form:

$$
-\rho \overline{u_{i}^{\prime} u_{j}^{\prime}}=\mu_{t}\left(\frac{\partial u_{i}}{\partial x_{j}}+\frac{\partial u_{j}}{\partial x_{i}}\right)-\frac{2}{3}\left(\rho \kappa+\mu_{t} \frac{\partial u_{i}}{\partial x_{i}}\right) \delta_{i j},
$$

where $\mu_{t}=\rho C_{\mu} \frac{k^{2}}{\varepsilon}$ based on the RNG $k-\varepsilon$ model, and $C_{\mu}$ equals 0.0845 , the transport equations for $k$ and $\varepsilon$ are listed as
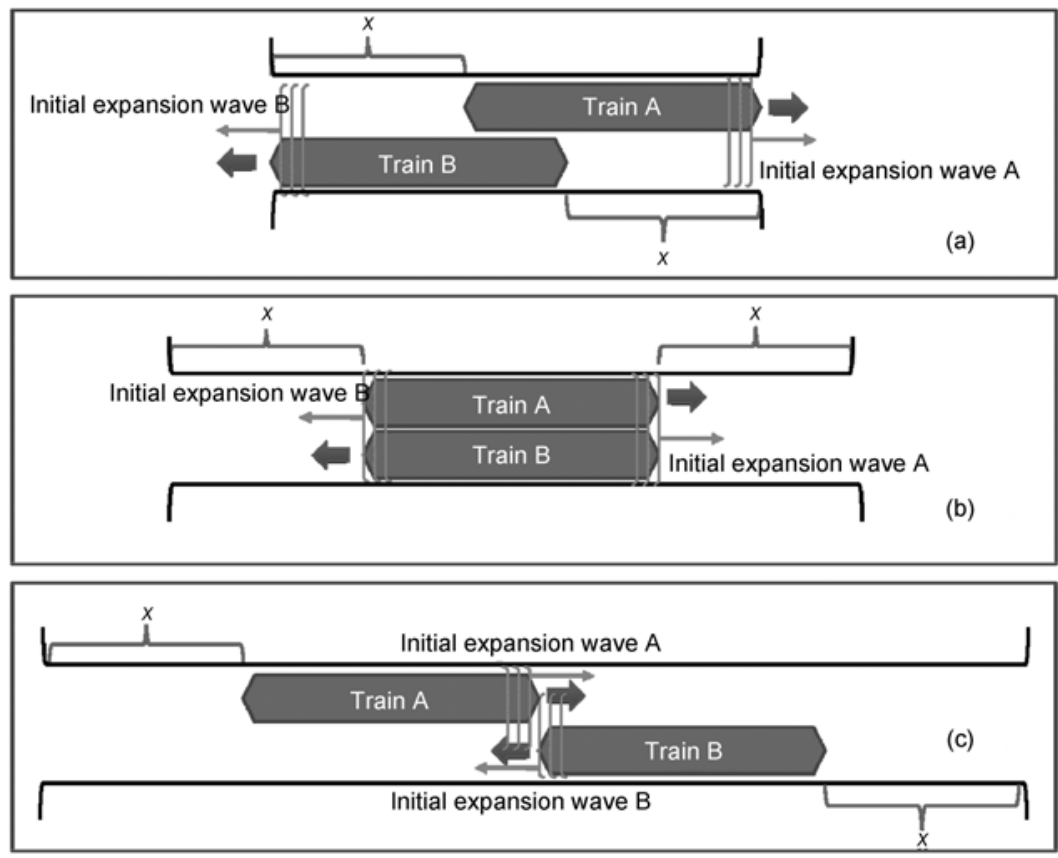

Figure 3 Development of pressure waves in tunnels with different critical lengths. 


$$
\begin{gathered}
\frac{\partial}{\partial t}(\rho k)+\frac{\partial}{\partial x_{i}}\left(\rho k u_{i}\right)=\frac{\partial}{\partial x_{j}}\left(\alpha_{k} \mu_{\mathrm{eff}} \frac{\partial k}{\partial x_{j}}\right) \\
+G_{k}+G_{b}-\rho \epsilon-Y_{M}+S_{k}, \\
\frac{\partial}{\partial t}(\rho \varepsilon)+\frac{\partial}{\partial x_{i}}\left(\rho \varepsilon u_{i}\right)=\frac{\partial}{\partial x_{j}}\left(\alpha_{\varepsilon} \mu_{\mathrm{eff}} \frac{\partial \varepsilon}{\partial x_{j}}\right) \\
+C_{1 \varepsilon} \frac{\varepsilon}{k}\left(G_{k}+C_{3 \varepsilon} G_{b}\right)-C_{2 \varepsilon} \rho \frac{\varepsilon^{2}}{k}-R_{\varepsilon}+S_{\varepsilon},
\end{gathered}
$$

where $\alpha_{k}, \alpha_{\varepsilon}$ are the reciprocals of effective Prandtl numbers of $k$ and $\varepsilon$, respectively.

$$
R_{\varepsilon}=\frac{C_{\mu} \rho \eta^{3}\left(1-\eta / \eta_{0}\right)}{1+\beta \eta^{3}} \frac{\varepsilon^{2}}{k}
$$

where $\eta \equiv S_{k} / \varepsilon, \quad \eta_{0}=4.38$ and $\beta=0.012$.

The eddy viscosity efficient satisfys the following relationship:

$$
\mathrm{d}\left(\frac{\rho^{2} k}{\sqrt{\varepsilon \mu}}\right)=1.72 \frac{\tilde{v}}{\sqrt{\tilde{v}^{3}-1-C_{v}}} \mathrm{~d} \tilde{v},
$$

where $\tilde{v}=\mu_{\text {eff }} / \mu$ and $C_{v} \approx 100$.

Other constants are listed as

$$
C_{1 \varepsilon}=1.42, C_{2 \varepsilon}=1.68 \text {. }
$$

\subsection{Solution strategy and computing conditions}

The finite volume method is adopted to solve the above governing equations. To account for the relative movement between the trains, the multi-block approach is used for mesh generation. The square zone around the train is firstly meshed and then the grids in the outer region and in the tunnel are generated. Sliding mesh strategy is adopted to achieve the relative motion between different blocks. Besides, the dynamic layering method [17] is adopted to maintain the invariance of the grids in outer region. The whole mesh and grids around the train head are depicted in Figures 4 and 5.

In the simulation, the tunnel wall is stationary while the two trains are moving at the real speed. A moving wall condition with a speed of $300 \mathrm{~km} / \mathrm{h}$ is adopted for the trains, and

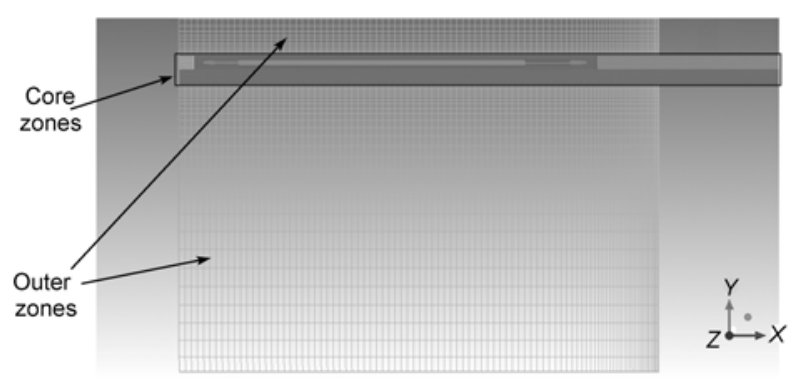

Figure 4 Global mesh of computation.

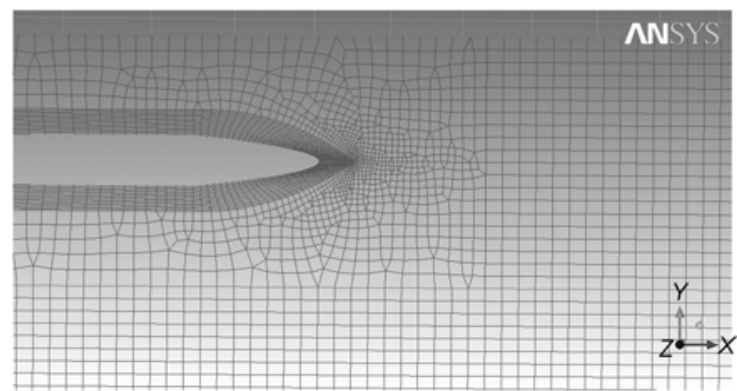

Figure 5 Grids near the head of train.

the stationary no-slip wall conditions are satisfied for the ground and tunnel wall. The far field pressure is set to be 1 atm. Probes on the leading car, the middle cars and the rear car are placed to record the pressure, and the highest and lowest pressures on the trains are recorded in every time step.

\section{Computational results}

\subsection{Comparison of different approaches}

A $2000 \mathrm{~m}$ tunnel case is firstly simulated with the approach mentioned above. The pressure contour when the train is entering the tunnel is shown in Figure 6, while as seen in Figure 7, the pressure drops in the flow field when the trains are passing each other. As Figure 8 shows, pressure histories on the typical probes on the leading and rear cars are obtained. For comparison, the similar case is performed with the online software Thermo-Tun [9] with results as Figure 9 shows, and a typical 3D result is shown in Figure 10. It can be seen that the pressure variations over time are similar for these 3 cases: In the initial stage, the maximum pressure appears on the leading car, and pressure does not rise on the rear car; when the initial expansion wave is in effect, the minimum pressure on the leading car is lower than on the rear; when the second expansion wave is in effect, there is still very low pressure appearing on the rear car, which is not obvious on the leading car. Due to the slightly differences in the head shapes, the blockage ratios and the locations of measuring points, the pressure amplitudes have a little difference. However, these parameters do not affect the characters and mechanism of pressure variation changing with tunnel length, which shows the feasibility of $2 \mathrm{D}$ simulation to verify the theoretical results.

\subsection{Comparison of results with different tunnel lengths}

Simulations of different tunnel lengths with the above method are performed, ranging from 500 to $2000 \mathrm{~m}$. A group of the maximum and minimum pressure histories over time on different positions are obtained. For example, the minimum pressure time histories on the leading car in tunnels with lengths from 600 to $800 \mathrm{~m}$ are drawn in Figure 11. Meanwhile, the minimum pressure time histories on the middle cars are drawn in Figure 12. 


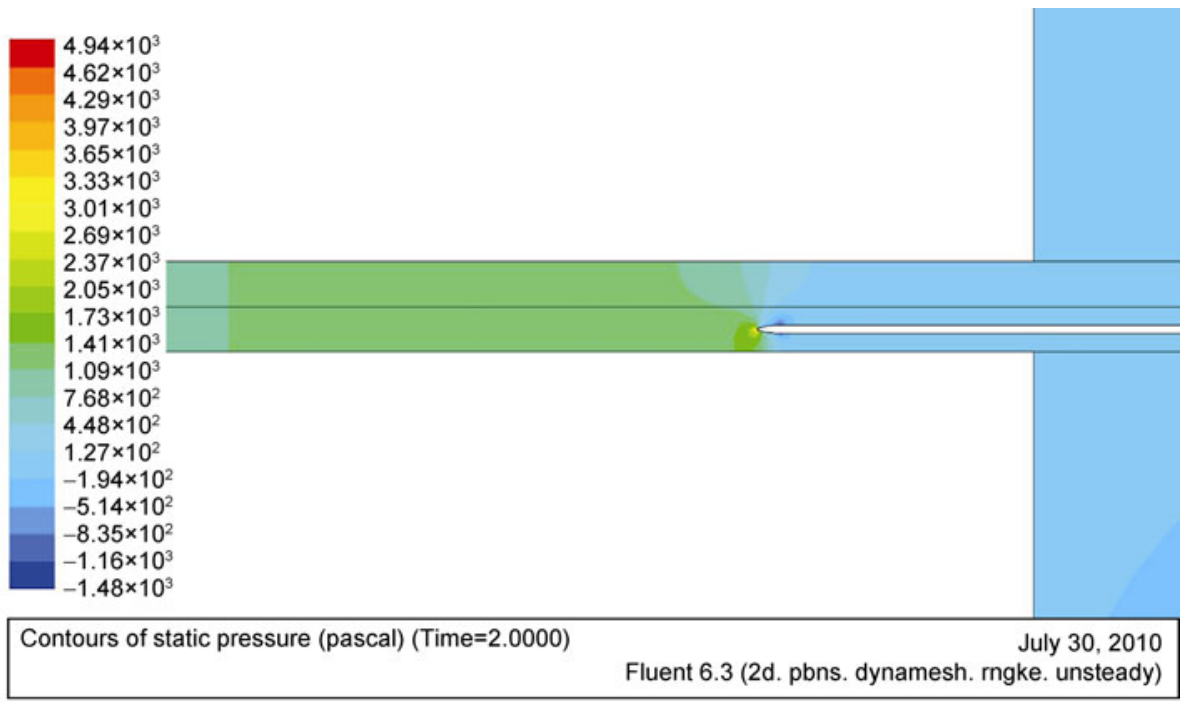

Figure 6 Top view of pressure contour when the train enters the tunnel.

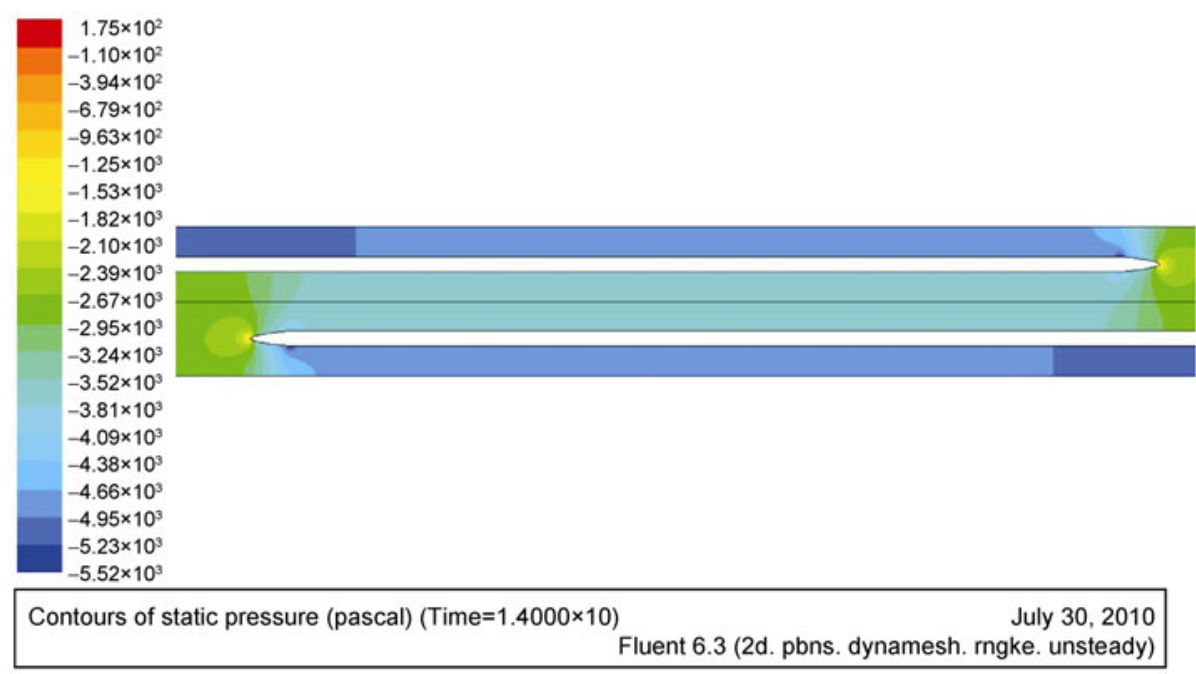

Figure 7 Top view of pressure contour when the trains pass by each other in the tunnel.

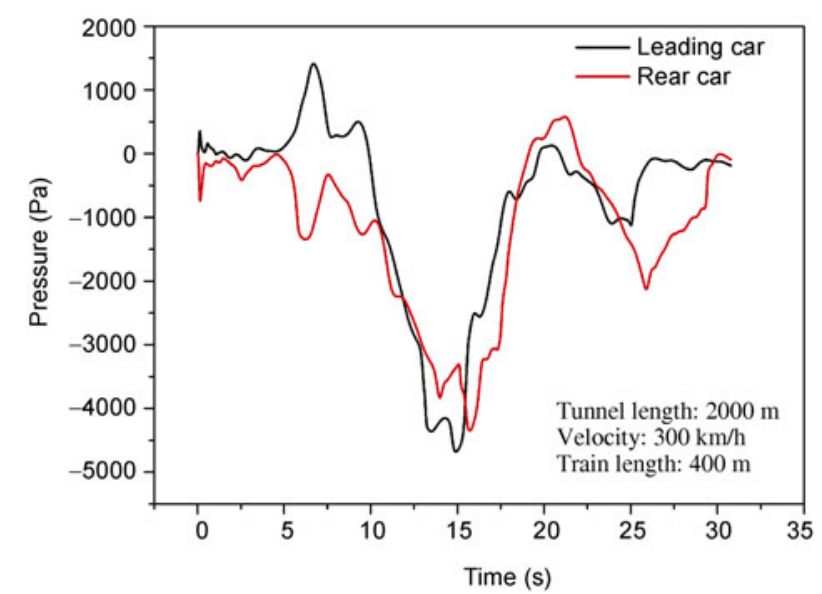

Figure 8 Pressure histories of typical measuring points obtained by 2D computation.

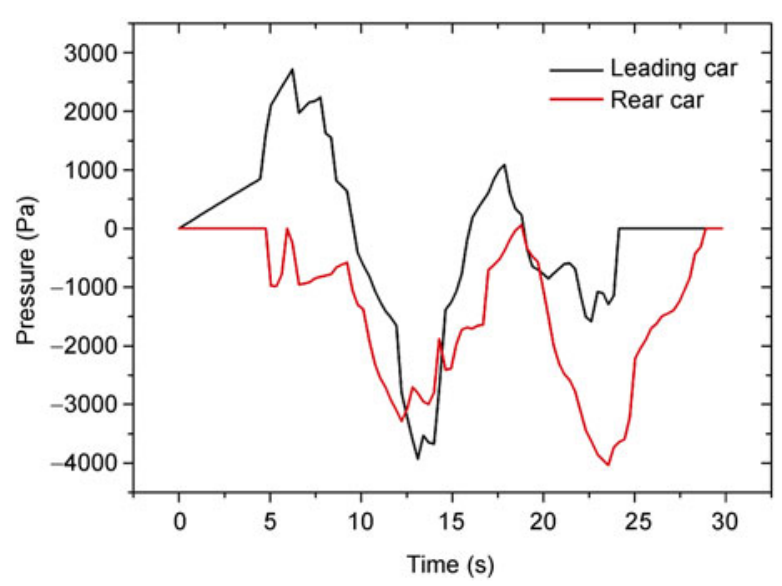

Figure 9 Pressure histories obtained by 1D computation in the software Thermo-Tun. 


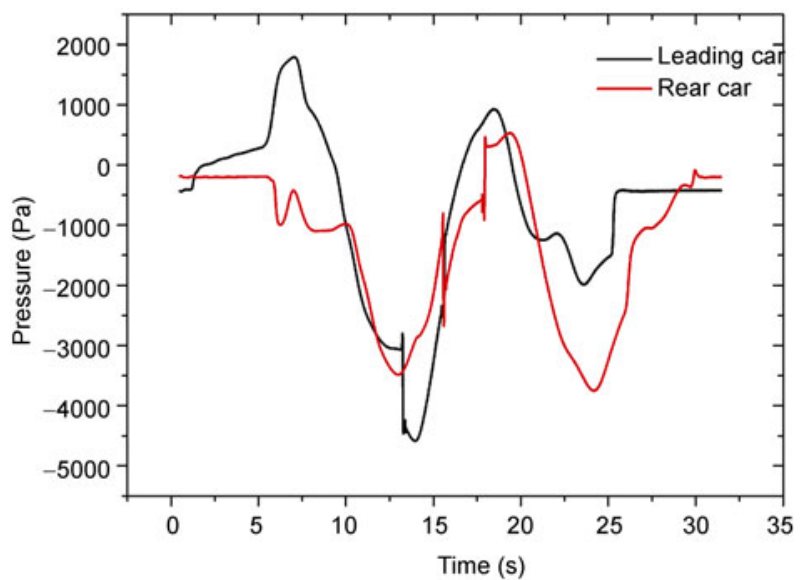

Figure 10 Pressure histories of typical measuring points obtained by 3D computation.

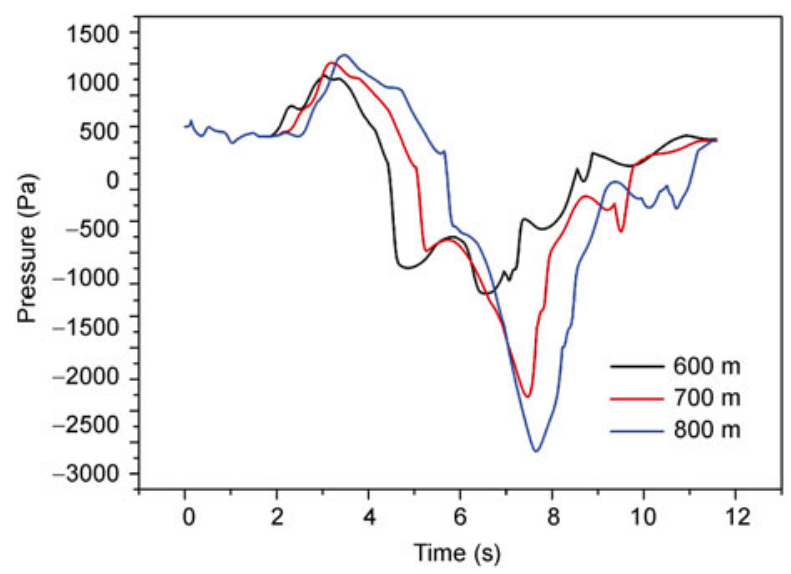

Figure 11 The histories of minimum pressure on the leading car in tunnels with lengths from 600 to $800 \mathrm{~m}$.

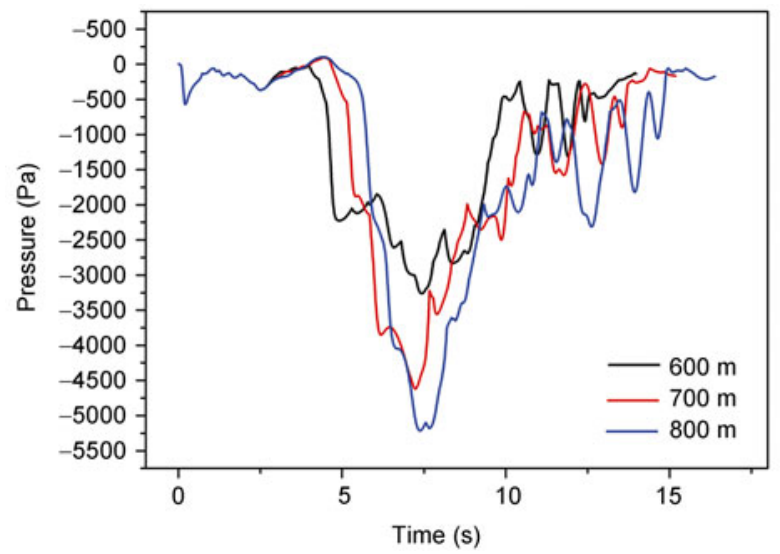

Figure 12 The histories of minimum pressure on the middle cars with lengths from 600 to $800 \mathrm{~m}$.

As Figures 13-17 show, the maximum and minimum pressures of different tunnel lengths are drawn as the same curve. The rear car is highly affected by the initial expansion waves, so there is no positive pressure that can be de-

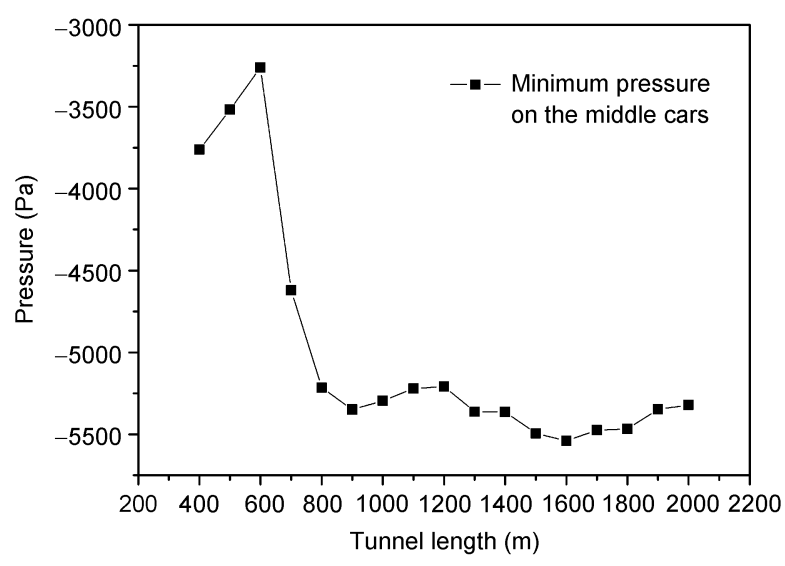

Figure 13 Global minimum pressures on the middle cars in tunnels with different lengths.

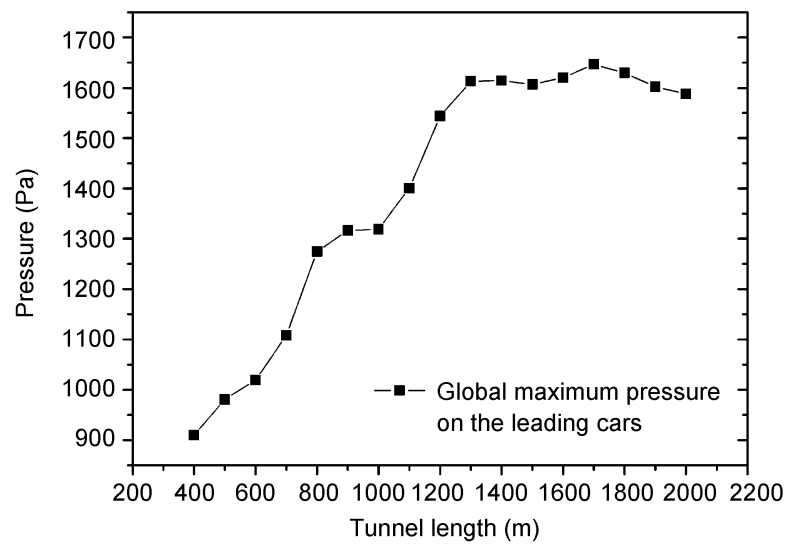

Figure 14 Global maximum pressures on the leading cars in tunnels with different lengths.

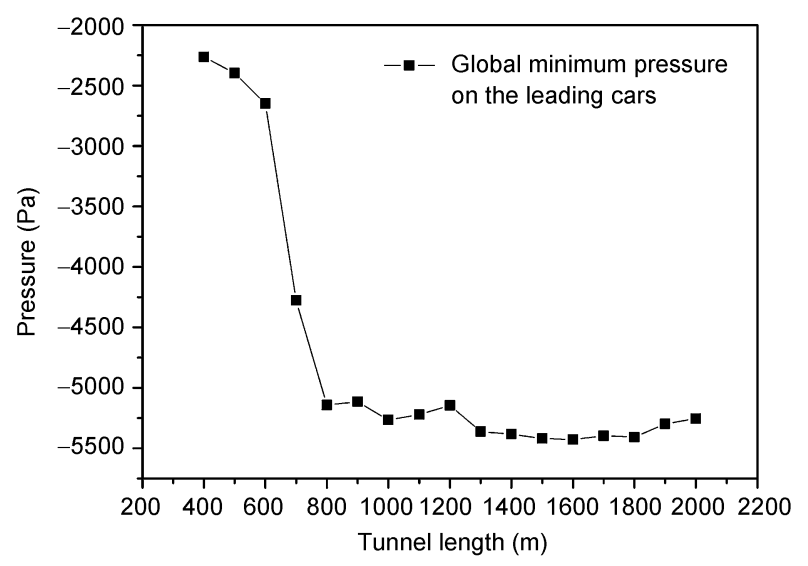

Figure 15 Global minimum pressures on the leading cars in tunnels with different lengths.

tected. Taking the minimum pressure on the middle cars as an example, the worst tunnel length exists at the length of $1600 \mathrm{~m}$, agreeing well with the earlier analysis.

Further investigation on Figures 14 to 17 reveals that the 


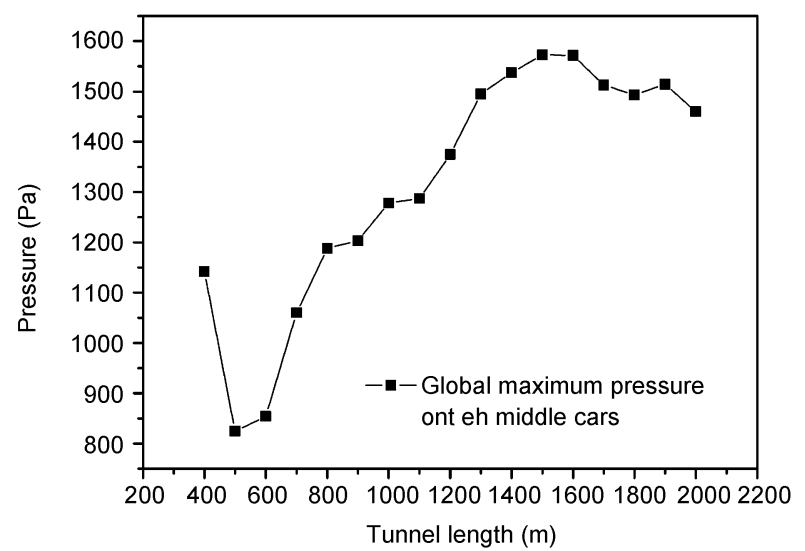

Figure 16 Global maximum pressures on the middle cars in tunnels with different lengths.

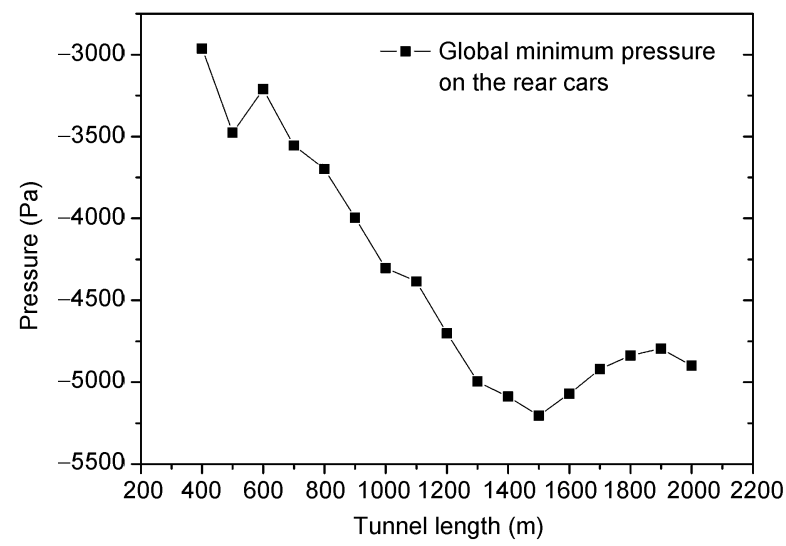

Figure 17 Global minimum pressures on the rear cars in tunnels with different lengths.

maximum pressure and the minimum pressure both arise near the length of $1632 \mathrm{~m}$, which verifies the result from theoretical analysis.

For the minimum pressure curves shown in Figures 15 and 17 , the value changes significantly in the tunnel length region from 500 to $1000 \mathrm{~m}$. This result accords well with the theoretical result that the critical length region for negative pressure is from 529 to $1060 \mathrm{~m}$.

\subsection{Pressure evolution in tunnel with the worst length}

In the condition that the tunnel length is the worst length $1600 \mathrm{~m}$, the pressure curve of a measurement point on the train head surface is shown in Figure 18 with typical time marked, and the contour of pressure field on typical time (shown in Figure 19) shows the followings.

1) When the front of the train already enters the tunnel, a series of compression waves are generated in front of the train and propagate forward. At this time the pressure waves have not reached the opposite train, therefore the measuring pressure almost does not change.

2) When the initial compression waves generated by the

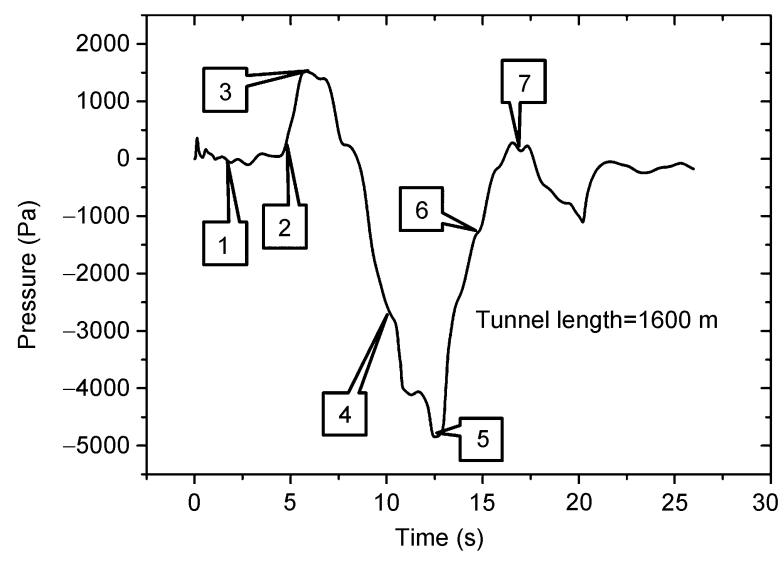

Figure 18 Pressure histories of typical measuring point on the leading car (the tunnel length is $1600 \mathrm{~m}$ ).

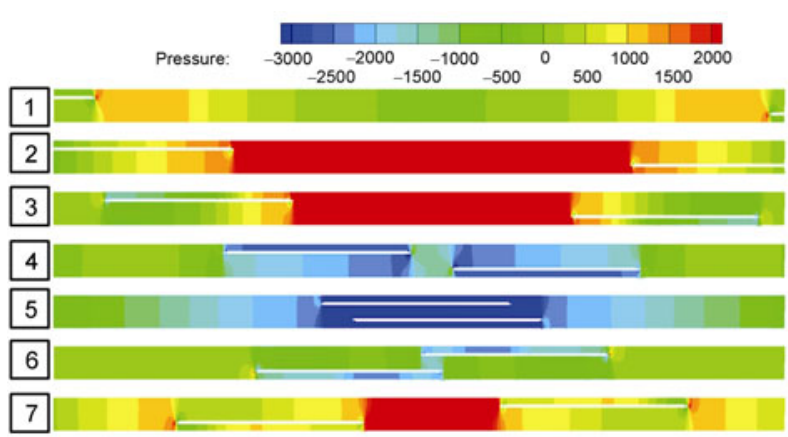

Figure 19 A time sequence of pressure contours (the tunnel length is $1600 \mathrm{~m})$.

opposite train propagate to the area around this train, the measuring pressure begins to rise.

3) When the initial compression waves generated by the opposite train propagate to the entrance of the tunnel, then reflect and change into expansion waves, and then superimpose with the initial expansion waves, the measuring pressure is at the highest point and begins to decrease.

4) When the front edge of superimposed expansion waves propagates past the measuring point, the measuring pressure decreases rapidly.

5) When the two trains pass by each other, the measuring pressure further drops to the minimum value.

6) When the two trains begin to leave each other, new compression waves are generated and induce the measuring pressure to recover gradually.

7) After the two trains completely leave each other, new pressure waves generated are slight, and pressure changes are not remarkable.

In this process, time differences between peak and valley pressure values are corresponding to the propagating time of pressure waves in the tunnel (about $4.7 \mathrm{~s}$ ). This can also explain that the propagating and reflecting of the pressure waves play the dominant role of the pressure variations on train and tunnel surfaces. 


\section{Conclusions}

The factors affecting the pressure waves are firstly theoretically analyzed, and the worst tunnel length corresponding to the maximum and minimum pressures is derived, which has a direct relationship between the length of the train and the running Mach number. For sixteen-coach marshalling trains with a speed of $300 \mathrm{~km} / \mathrm{h}$, the worst tunnel length is $1632 \mathrm{~m}$. Meanwhile the critical length region in which the minimum pressure changes significantly is deduced. Results show that if tunnel length is greater than $1060 \mathrm{~m}$, the negative pressure on train surfaces will be severe.

Then the two-dimensional approach is adopted to simulate trains passing by each other with different tunnel lengths. The worst tunnel length is around $1600 \mathrm{~m}$, and the minimum pressure varies effectively between 500 and $1000 \mathrm{~m}$, which greatly validate the result from theoretical analysis.

The formulas in the present work are dimensionless and have very straightforward forms, which can be easily applied to the design of real tunnels and the train-tunnel simulators. Besides, it could be easily extended to the case of two trains passing by each other in the tunnel with different tunnel-entering times, and the maximum pressure under the worst tunnel length condition should be close to the result given in the present work.

This work was supported by the National Key Technology R\&D Program, Aerodynamic Optimization Design and Safe Evaluation Techniques on Chinese High-Speed Trains (Grant No. 2009BAG12A03) and the National Basic Research Program of China ("973" Project) (Grant No. 2011CB71100).
1 Schetz J A. Aerodynamics of high-speed trains. Annu Rev Fluid Mech, 2001, 33: 371-414

2 Raghunathan R S, Kim H D, Setoguchi T. Aerodynamics of high-speed railway train. Prog Aerosp Sci, 2002, 38: 469-514

3 Tian H. Train Aerodynamics. Beijing: China Railway Publishing House, 2007

4 Mei Y, Zhou Z, Xu J. Tunnel Aerodynamics of High-speed Railway. Beijing: Science Press, 2009. 206-208

5 Wang J, Wan X, Wu J. Influence of tunnel lengths upon air pressure fluctuation in high speed railway tunnels. Modern Tunnel Tech, 2008, 45(6): $1-4$

6 Wan X, Wu J. In-situ test and study on the aerodynamic effect of the rolling stock passing through tunnels with a speed of $200 \mathrm{~km} / \mathrm{h}$. Modern Tunnel Tech, 2006, 43(1): 43-48

7 Baker C J, Dalley S, Johnson J, et al. The slipstream and wake of a high-speed train. Proc Inst Mech Eng Prat F, 2001, 215: 83-99

8 Zhao W, Gao B, Wang Y. Analysis of the pressure wave variation generated by a high- speed train passing through a tunnel. Modern Tunnel Tech, 2004, 41(6): 16-19

9 Thermo Tun-Software. http://www.thermotun.com/index.htm

10 Jiang Z, Matsuoka K, Sasoh A, et al. Numerical and experimental investigation of wave dynamic processes in high-speed train/tunnels. Acta Mech Sinica, 2002, 18(3): 209-226

11 Ricco P, Baron A, Molteni P. Nature of pressure waves induced by a high-speed train travelling through a tunnel. J Wind Eng Ind Aerod, 2007, 95: 781-808

12 Chen R L, Zeng Q Y, Zhong X G, et al. Numerical study on the restriction speed of train passing curved rail in cross wind. Sci China Ser E-Tech Sci, 2009, 52(7): 2037-2047

13 Sun Z X, Song J J, An Y R. Optimization of the head shape of the Crh3 high speed train. Sci China Tech Sci, 2010, 53(12): 3356-3364

14 Wang Y W, Wang Y, An Y R, et al. Aerodynamic simulation of high-speed trains based on the Lattice Boltzmann Method (Lbm). Sci China Ser E-Tech Sci, 2008, 51(6): 773-783

15 Howe M S. On the infrasound generated when a train enters a tunnel. J Fluid Struct, 2003, 17: 629-642

16 Zhao W, Gao B, Zhang Z. Three-dimensional numerical simulation of high-speed trains passing through tunnel. China Railway Sci, 2003, 24(5): 96-100

17 Fluent Inc. FLUENT 6.3 User's Guide. http://www.fluent.com 